Wave coupling in the magnetized plasma edge: Impact of a finite, inhomogeneous density inside the antenna box

L. Lu' , K. Crombé, D. Van Eester, L. Colas, and J. Jacquot

Citation: AIP Conference Proceedings 1689, 050003 (2015); doi: 10.1063/1.4936491

View online: http://dx.doi.org/10.1063/1.4936491

View Table of Contents: http://aip.scitation.org/toc/apc/1689/1

Published by the American Institute of Physics 


\title{
Wave coupling in the magnetized plasma edge: impact of a finite, inhomogeneous density inside the antenna box
}

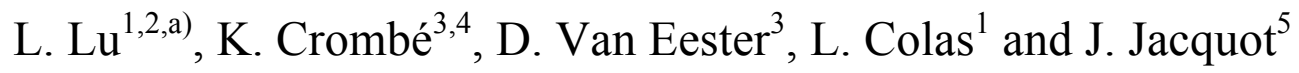 \\ ${ }^{I}$ CEA, IRFM, F-13108 Saint-Paul-Lez-Durance, France \\ ${ }^{2}$ Institut Jean Lamour, University of Lorraine, Nancy, France \\ ${ }^{3}$ EURATOM-Belgian State, LPP-ERM-KMS, TEC partner, Brussels, Belgium \\ ${ }^{4}$ Department of Applied Physics, Gent University, Belgium \\ ${ }^{5}$ Max-Planck-Institut für Plasmaphysik, EURATOM-Assoziation, Garching, Germany.
}

${ }^{a)}$ Corresponding author: ling-feng.lu@cea.fr

\begin{abstract}
Most present Ion Cyclotron Resonant Frequency (ICRF) heating codes and antenna codes assume the antenna sitting in a vacuum region and consider the fast wave only, which implicitly performs an abrupt density transition from vacuum to above lower hybrid $(\mathrm{LH})$ resonance. We studied the impact of densities that decay continuously inside the antenna box on near field patterns and power coupling. A new full wave code based on the COMSOL Finite Element Solver has been developed to investigate this topic. It is shown that: up to the memory limits of the adopted workstation, the local RF field pattern in low-density regions below the LH resonance changes with the grid size. Interestingly and importantly, however, the total coupled spectrum is independent to the mesh size and is weakly affected by the presence of the density profile inside the antenna box in dipole phasing. Thus one can drop out this density for coupling studies. Simulation also shows that varying the density gradient in the fast wave evanescence region has no significant effect on wave coupling.
\end{abstract}

\section{INTRODUCTION}

In an ion cyclotron resonant heating system, waves are excited by straps. The orientation of the straps is chosen so that they mainly excite the fast wave. The fast wave can propagate into high density plasma so it is often used as heating wave. The other polarization mode under cold plasma approximation, the slow wave can propagate in plasma with fairly low densities. This branch of wave is essential in sheath formation [1]. Most present ICRF heating codes and antenna codes $[2,3]$ assume the antenna sitting in a vacuum region and consider the fast wave only, whereas in reality a low but finite density is actually present inside the antenna box and a fast wave antenna parasitically excites the slow wave. So an intriguing question is whether this presence of density profile inside the antenna will significantly change the results. Things become more complicated when a special layer called lower hybrid (LH) resonance [4] appears inside the plasma filled-in antenna box. The place where the slow wave has its $\mathrm{LH}$ resonance is defined by $\mathrm{S}=0$, where $\mathrm{S}$ is the perpendicular diagonal component of the Stix dielectric tensor [5]. For typical tokamak parameters, it appears when the plasma density approaches $10^{17} \mathrm{~m}^{-3}$. The density is well beyond the LHR at the last closed flux surface but well below it or marginally below it at the position of the straps. Hence the LH resonance is a natural ingredient of wave behavior close to launching structures. The main question being whether the power carried by the slow wave to this resonance is significant or not. The wavelength of the slow wave shrinks to zero at this resonance. One may wonder if it is possible to numerically capture this wave sufficiently accurately. And if it is not, does a non-converged result inside the antenna box significantly affect the results outside the box? In this paper, we answered this question for the key parameter, the coupling power. 


\section{DESCRIPTION OF 2D FULL WAVE COUPLING CODE WITH TILTED B}

A 2D full wave coupling code with tilted magnetic configuration [6,7] dedicated to study wave coupling problems in a collisionless magnetized cold edge plasma has been developed and tested based on COMSOL Multiphysics.

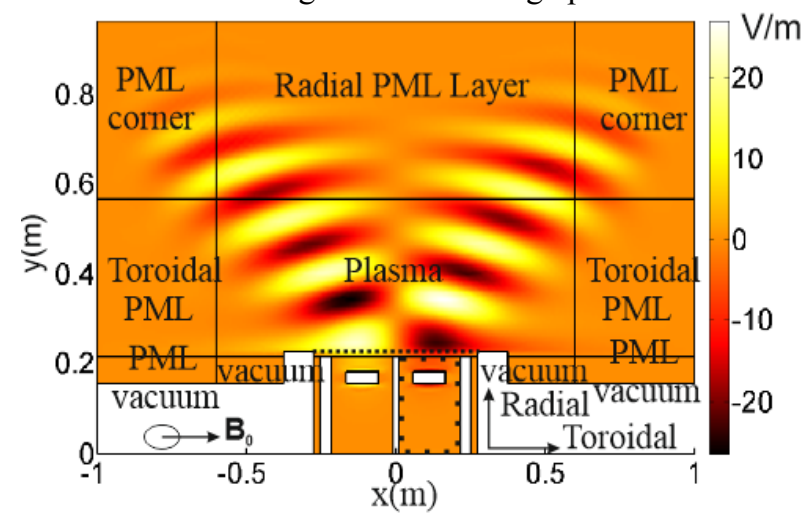

FIGURE 1. Schematic view of 2D full wave coupling code with tilted $\mathbf{B}_{\mathbf{0}}$. The color indicates the radial E field
The model has a realistic $2 \mathrm{D}$ geometry, shown in FIGURE 1, where the $\mathrm{x}$ direction in COMSOL corresponds to the toroidal direction, $\mathrm{y}$ is the radial direction and $\mathrm{z}$ is the (out of plane component) poloidal direction in a Tokamak geometry where the curvature effect has been ignored. The magnetic field lies in $\mathrm{x}-\mathrm{z}$ plane. Its direction is characterized by tilt angle theta $(\theta)$, which is the angle between field line and the $\mathrm{x}$ direction. The antenna includes two straps, the antenna box, central septum and lateral limiters, indicated as the blank components. The antenna aperture is shown as a dash line. Perfect Matched Layer (PML) technique described in [6] are extended to tilted $\mathbf{B}_{0}$ and being used to mimic purely outgoing waves emanating to infinity (single pass absorption). The cold plasma is described by the Stix dielectric tensor, which is rotated

under the same convention as the magnetic field in the code. In this $2 \mathrm{D}$ code, the poloidal geometry is infinite and homogenous. So the poloidal derivative is by default set to zero. The excitation, dipole or monopole phasing is done by imposing uniform co-directional, or counter-directional real currents on 2 straps in the $\mathrm{z}$ direction. All the simulations are done with 1 A current on each strap.

In this paper we use a plasma solely composed of D ions. Furthermore, the following Tore Supra like parameters are taken [7]: magnetic field at aperture $\mathbf{B}_{0}(\mathrm{y}=0.224)=2.32 \mathrm{~T}, \mathrm{RF}$ wave frequency $48 \mathrm{MHz}$. The magnetic field strength scales as $1 / R$, with $R$ major radius axis. A set of continuous density profiles is used inside the antenna box. Density 1 takes the experimental density distribution (coming from the reflectometer) in the main (edge) plasma and extrapolates a constant value $10^{17} \mathrm{~m}^{-3}$ in the antenna box. Densities 2-4 follow the same profile as density 1 in the main plasma, but add an exponential decay inside the antenna box with a reference density $10^{18} \mathrm{~m}-3$ at $\mathrm{y}=0.2195 \mathrm{~m}$. Density 2: $\mathrm{n}_{\mathrm{e}}=10^{18} \exp (-(0.2195-\mathrm{y}) / 0.0933)$, density $3: \mathrm{n}_{\mathrm{e}}=10^{18} \exp (-(0.2195-\mathrm{y}) / 0.0477)$ and density $4: \mathrm{n}_{\mathrm{e}}=10^{18} \exp (-$ $(0.2195-\mathrm{y}) / 0.0318)$. All the density profiles are homogeneous in toroidal direction. Density 1 and 2 are above LH resonance, whereas LH resonance is crossed both on density 3 and density 4 . It locates at $y=0.1056 \mathrm{~m}$ and $\mathrm{y}=0.1434 \mathrm{~m}$ respectively.

\section{NON CONVERGED FIELDS BELOW LOWER HYBRID RESONANCE}

FIGURE 2 plots the $\mathbf{E}$ field in the right half box (surrounding by dots in FIGURE 1). It uses the exponential density profile 3 and 4 with the same mesh 2, which has $52 * 108$ grid points. Locations of the lower hybrid resonance are depicted by solid line. One can see below the resonance, new fields start to grow and as the density decreases, more and more modes appear. In dipole phasing, this structure is anti-symmetric with respect to the antenna septum,

under the condition of symmetrical mesh. In this region, the radial electric field is about 10 times larger than the other 


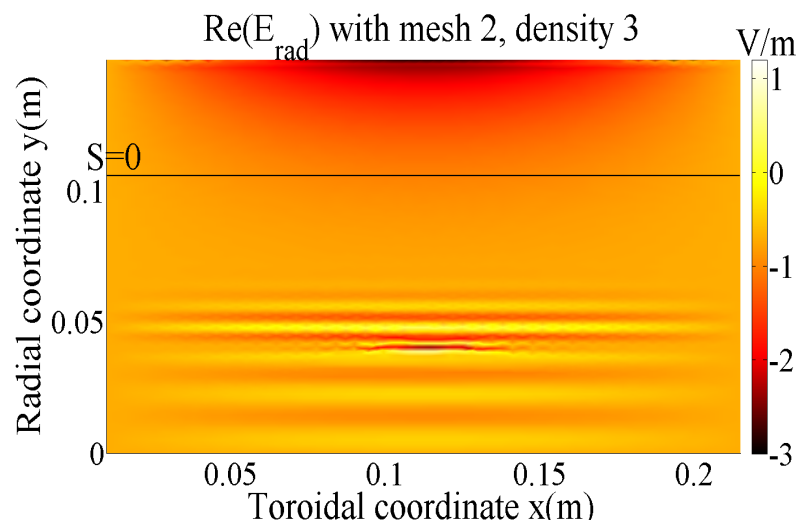

(a)

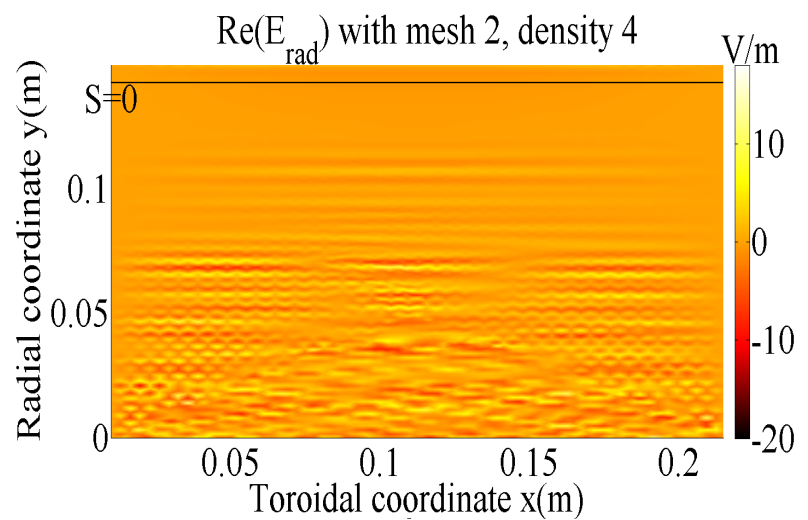

(b)

FIGURE 2. Radial electric field with different density profiles, but the same mesh. Dipole phasing, theta $=0^{0}$ two components. The slow wave propagates between the LH resonance $(S=0)$ and $S=n_{/ /}^{2}$, where the parallel refractive index $\mathrm{n}_{/ /}$is determined by the antenna geometry and phasing, which can approximately be seen as an invariant spectrum. From the slow wave dispersion relation, one knows that in its propagating region, for a given $k_{/ /}$, the perpendicular wave vector $k_{\perp}$ is decreasing as the density decreases. This is consistent with FIGURE 2 (a), where the $\lambda_{\perp}$ is increasing as the density decreases from upper to bottom. Comparing (b) with (a), one can see that when the density switches from density 3 to density 4 , more modes with lower $\mathrm{k}_{/ /}$, thus larger $\lambda_{/ /}$become propagating. This can be understood from $k_{\perp}^{2}=k^{2}-k_{/ /}^{2}$, when the density decreases, both $k_{\perp}^{2}$ and $k^{2}$ are decreasing, so in order to have a positive $k_{\perp}^{2}$, one needs a smaller $k_{/ /}$. Aside from propagating along radial direction, from FIGURE 2 (b), one can see the modes bounce between two toroidal metallic walls, like cavity modes, where modes with $k_{/ /}=\frac{2 \pi n}{L}$ (with $\mathrm{n}$ an arbitrary integer and $\mathrm{L}$ the toroidal dimension of metallic box) are added up together. It's not a lossless resonant cavity for the slow wave. The incident slow wave can be mode converted to the fast wave at the wall in order to fulfill the metallic boundary condition under tilted $\mathbf{B}_{\mathbf{0}}$ [8] or at the LH resonance. It thus transfers the wave energy from the slow wave to the fast wave. Since there is no damping mechanism considered, under current density profiles, finally all the radiative energy (real part of power flow) should be transferred to the fast wave, which then carries this power out of the antenna box and only remains the slosh power (imaginary part of power flow) bouncing inside box.

Plotting FIGURE 2 with different meshes gives significantly different field structure and scale. A further test shows the non-convergence persists up to the memory limits (64GB RAM) of the adopted workstation. This nonconvergence behavior of the field below LH resonance has been independently observed by another finite difference code [9]. Below the $\mathrm{LH}$ resonance, the slow wave is propagating. From its dispersion relation, a wave with high $\mathrm{k}_{/ /}$, is more likely to propagate. As a consequence, the slow wave having a nature of short wavelength can be very sensitive to the mesh size. While the slow wave also has a very short evanescence length, the electric field out of this lower hybrid region is not affected by this non-convergence problem.

Under large tilt angle, the antenna is more likely to excite the slow wave, which will cause numerical instability. Indeed when we increase the tilt angle from theta $=0^{0}$ to theta $=60^{\circ}$. The magnitude of the difference of radial electric field between two different meshes is increasing. Phasing has less influence on the field structure. We compare FIGURE 2 with its counterpart in monopole phasing. The radial field below the antenna box shows the same structure and only has a relative error $2 \mathrm{e}-3 \mathrm{~V} / \mathrm{m}$ in density 3 and $2 \mathrm{e}-2 \mathrm{~V} / \mathrm{m}$ in density 4 . The mesh size near the wall is also important. The electric field in FIGURE 2 (b) changes up to $200 \%$ after adding a specific mesh refinement in the region within a distance of $0.008 \mathrm{~m}$ to the antenna box wall. 


\section{POWER COUPLING INDEPENDENT ON THE MESH, LESS SENSITIVE TO THE PRESENCE OF PLASMA AND DENSITY GRADIENT}

The power emanating from strap is denoted as,

$$
-\frac{1}{2} \operatorname{Re}\left(\int_{V} \mathbf{E} \cdot \mathbf{J}^{*} d V\right)=\operatorname{Re}(\mathbf{P})
$$

Since we only imposed poloidal current, the LHS can be reduced to a line integral to a scalar product. FIGURE 3 shows poloidal electric field is independent of the mesh, which guarantees the radiating power independence of the mesh. Physically this is because the slow wave only carries slosh power.

Most antenna design codes assume the antenna is in vacuum. It is interesting and important to check how different the power and electric field near the antenna aperture are when one compares the difference between a plasma filled-in and a vacuum antenna box. FIGURE 4 shows a power variation in a

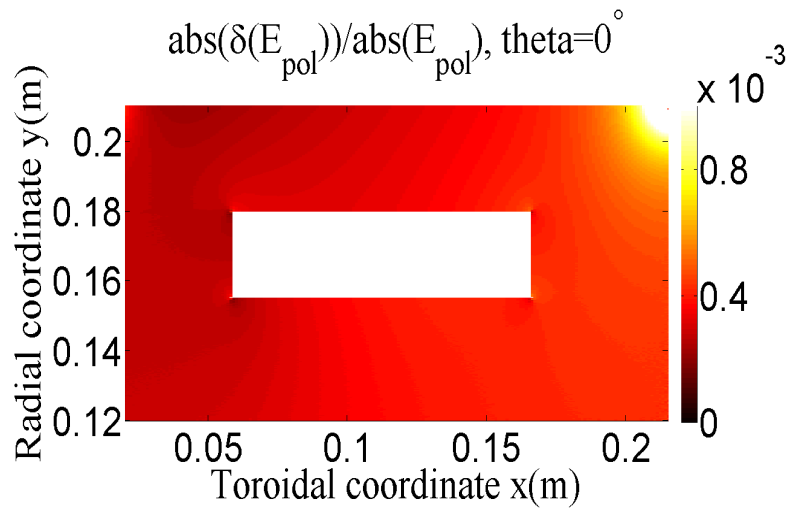

FIGURE 3. relative error of poloidal electric field surrounding the strap under two different meshes, tilt angle $0^{\circ}$

scan of tilt angle under mesh 2. The power is calculated by doing

a line integration of radial power flow across the antenna aperture. For dipole phasing, the power looks independent of whether the antenna is in plasma or in vacuum. For monopole phasing, there is maximally $20 \%$ difference between those two cases. The power level in monopole phasing is higher than in dipole phasing, this is expected since the evanescence region for the fast waves is shorter in monopole phasing than in dipole.

The fast wave $R$ cut-off layer, calculated from Eq. (2) [7] is well above the aperture in dipole phasing. However, in monopole phasing, the $R$ cut-off layer is generally inside the antenna box, thus the fast wave evanescence region is larger in a vacuum antenna than in a plasma filled in antenna. Consistent with this, the spectrum has a drop in low $k_{/ /}$when one switches from a plasma filled in antenna to a vacuum antenna. This explains the $20 \%$ of power difference mentioned before.

From the fast wave dispersion relation, one can derive the expression for the $R$ cut-off location [7],

$$
n_{x}^{2} \sin ^{2} \theta=\frac{\left(n_{x}^{2} \cos ^{2} \theta-R\right)\left(n_{x}^{2} \cos ^{2} \theta-L\right)}{S-n_{x}^{2} \cos ^{2} \theta}
$$

Where $\mathrm{n}_{\mathrm{x}}$ is the toroidal reflective index. $\mathrm{R}$ and $\mathrm{L}$ from the Stix tensor. We choose a value of 9.14 in the calculation, which is the main lobe of power spectrum.

Dipole phasing is used as the main heating phasing [10]. For

Power flow across antenna aperture

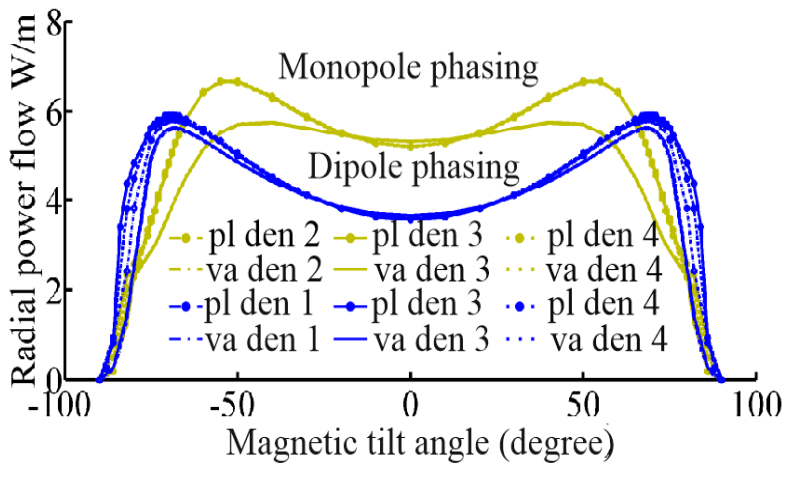

FIGURE 4. Radial power flow across aperture. pl (plasma), va(vacuum), den (density)

this phasing, the effect of density gradient in the fast wave evanescence region on power coupling is also studied. We tested three tilt angles, i.e. theta $=0^{0}, 7^{0}$ and $20^{0}$.For each tilt angle, calculate the $R$ cut-off location using Eq. (2). Then starting from the $R$ cut-off, a set of exponential density profiles with different decay lengths is imposed. Results show that changing density gradient in the fast wave evanescence region can maximally leads to $5 \%$ of power variation.

\section{CONCLUSION}

The presence of plasma inside the antenna box can make numerical simulation more problematic when the density crosses the LH resonance. Up to the memory limits of the dedicated workstation, fields below the LH resonance is not converged due to the short wavelength of the slow wave. It's critical to be aware of this near field 
non-convergence when studying near field effect inside the antenna box. With a dominant poloidal current imposed on the straps, it is shown that the poloidal electric field and thus the total excited power is independent of the mesh, which guarantees the power transmitted to the main plasma is also independent of the mesh or the non-convergence property of the fields inside the antenna box. All the radiating power carried by the slow wave is transferred to the fast wave. In dipole phasing, the total power coupled to main plasma is indifferent with the plasma density inside the antenna box so that one can drop out the low density inside the antenna box when studying coupling issues. In monopole phasing, simulation shows there is a maximum $20 \%$ of power increase due to the presence of plasma. The distinction comes from the fact that the fast wave evanescence length for low $k_{/ /}$is changing. Hence modeling low $k_{/ /}$ scheme with antenna staying in vacuum may need to be re-considered. The present model does not consider parasitic damping and sheath effects. The poloidal derivative which we ignored is important in studying the density modification by ponderomotive forces [11]. Those effects will be left for the future study. The density measurement in the fast wave evanescence region is affected by the largest errors [12]. This is again crucial in determining near field. Nevertheless, changing density gradient in the evanescence region can maximally affect power coupling by $5 \%$. One can thus still model the power coupling to plasma in a fairly well precision despite some uncertainties in density profile.

Acknowledgments: This work has been carried out within the framework of the EUROfusion Consortium and has received funding from the Erasmus Fusion-DC and the Euratom research and training programme 2014-2018 under grant agreement No 633053. The views and opinions expressed herein do not necessarily reflect those of the European Commission.

\section{REFERENCES}

1. J. Jacquot et al, Phys. Plasma 21, 061509 (2014) 7. L. Lu, L. Colas, Private communication (2013)

2. V. Lancellotti et al, Nucl. Fusion 46(2006) 8. D. A. D'Ippolito et al, Phys. Plas, 15, S476-S499 102501(2008)

3. A. Messiaen et al, Nucl. Fusion 50025026 9. K.Crombé et al, AIP conf. proc. 1580, 318-321 (2010)

4. P. M. Bellan, Phys. Plasma 1(11), Nov. 1994

5. T.H.Stix, waves in plasmas, AIP Press (1992) (2014)

6. J. Jacquot et.al, PPCF 55115004 (2013)

10. A. Messiaen et al, PPCF, 53 (2011) 085020

11. D. Van Eester et al, PPCF, 55025002 (2013)

12. D. Milanesio, et al, PPCF, 55045010 (2013) 